\title{
MODELADO DEL REGISTRO AUTOMATIZADO DE LA GESTIÓN DOCENTE PARA UNA INSTITUCIÓN UNIVERSITARIA
}

\author{
María Geizzelez Luzardo ${ }^{1 *}$ \\ ${ }^{1}$ Universidad del Zulia. Maracaibo, Venezuela \\ *Autor de correspondencia: mgeizzelez@gmail.com
}

Recibido Febrero 2020; Aceptado Mayo 2020

\begin{abstract}
Resumen - El propósito de este trabajo consiste en diseñar una base de datos para un sistema automatizado de gestión docente, tomando como referencia las experiencias en la Escuela de Nutrición y Dietética de LUZ, a fin de automatizar los procesos relacionados con el registro e inspección de cada una de las actividades docentes que se ejecutan dentro de esta instancia universitaria. Para ello se empleó el método de Ciclo de Vida del Desarrollo de Sistemas propuesto por los autores Kendall y Kendall (2011) hasta la fase de diseño. Para la recolección de datos se utilizó la observación directa con la finalidad de definir los requerimientos que debía cubrir el sistema, así mismo se evidenció que el proceso de registro de actividades docentes no alcanzaba las expectativas demandadas, puesto que éstas se realizan de forma manual lo que genera pérdida de tiempo y errores de cálculo en el suministro de la información, por lo cual se llegó a la conclusión de automatizar dicho proceso mediante un sistema de información que proporcione soluciones inmediatas a los requerimientos relacionados con el proceso de gestión docente de la institución, a fin de manejar con mayor confiabilidad y eficacia toda la información a procesar por el mismo.
\end{abstract}

Palabras clave: Automatización, sistema, gestión docente, base de datos, modelado de datos.

\section{MODELING OF THE AUTOMATED RECORD OF TEACHING MANAGEMENT FOR A UNIVERSITY INSTITUTION}

\begin{abstract}
The purpose of this work consists of designing a database for an automated system of teaching management, taking like reference the experiences in the School of Nutrition and Dietetic from LUZ, in order to automate the processes related to the registry and inspection of each one of the educational activities that are executed within this university instance. For this purpose, the System Development Life Cycle method proposed by the authors Kendall and Kendall (2011) was used to the phase of design. For data collection used direct observation with purpose to define requirements that had to cover the system, also demonstrated that process of registry of activities educational not reached expectations demanded, since these are made of manual form what generates lost of time and errors of calculate in the provision of the information, thus reached the conclusion to automate this process by means of an information system that provides solutions immediate to the requirements related to the process of educational management of the institution, in order to handle with greater trustworthiness and effectiveness all the information to process by the same one.
\end{abstract}

Keywords: Automatization, system, teaching management, database, data model. 
Modelado del registro automatizado de la gestión docente para una institución universitaria

\section{Introducción}

Los avances en el ámbito de las nuevas tecnologías de información y comunicación han contribuido al desarrollo de herramientas que aporten alternativas de solución a problemas presentes en las organizaciones en lo que respecta al procesamiento de información y toma de decisiones. En este sentido, los sistemas automatizados han desempeñado un rol sumamente importante al procesar y producir de manera confiable y precisa la información, ofreciendo soluciones a problemas organizacionales e institucionales, brindando a los usuarios una plataforma de trabajo acorde a sus necesidades y requerimientos (Linares \& Geizzelez, 2016; Miranda et al., 2016; Romero \& Quintero, 2014).

Sin embargo, existen instituciones que poseen sistemas de información mas no cumplen con todas las exigencias requeridas por los procesos implementados en ellos (Jiménez et al., 2016; Castillo et al., 2015); situación que se evidencia en la Secretaría Docente de la Escuela de Nutrición y Dietética de la Universidad del Zulia (LUZ) donde los procesos de gestión docente se llevan mediante el uso de formatos (de evaluación del curso, control de gestión docente, control de actividades académicas semestrales, informes del profesor, horarios académicos y permanencia) que son llenados manualmente por los docentes de los diferentes departamentos de la escuela y luego son entregados al jefe del departamento para que en la asamblea departamental sean discutidos al comienzo de cada periodo semestral.

Esta situación genera pérdida de tiempo al momento de realizar el proceso de evaluación, errores de cálculo al evaluar la información suministrada por los estudiantes al profesor, aunado a la inexistencia de registros que permitan realizar una comparación de la gestión docente de un semestre con respecto a otro período, trayendo como consecuencia fallas en el control de ciertos parámetros e indicadores de la actividad académica que, al no estar disponibles en cualquier momento que se requieran, no facilitan ni apoyan el proceso de toma de decisiones a objeto de generar estrategias de solución adecuadas.

De igual manera, la Secretaría Docente de la escuela adolece de informes por períodos que le permitan supervisar las actividades de los docentes adscritos a cada departamento. Por ello, se plantea el diseño de una base de datos para un sistema de información automatizado que proporcione soluciones inmediatas a los requerimientos que estén relacionados con el proceso de gestión docente de la institución, a fin de manejar con mayor confiabilidad y eficacia toda la información a procesar por el mismo, ofreciendo al personal docente la oportunidad de disponer de la tecnología que podrá ofrecer en un mínimo de tiempo toda la información que requieran las diferentes instancias universitarias, con un alto promedio de exactitud y confiabilidad en cuanto al proceso de gestión docente (Fong et al., 2019; Acosta et al., 2017; Morell, 2017; Santana et al., 2017; Barrera, et al., 2017; Tigre et al., 2016).

En tal sentido, se plantea como objetivo general del artículo diseñar una base de datos para el registro automatizado de la información generada en el proceso de gestión docente en la Escuela de Nutrición y Dietética de LUZ. Para alcanzar dicho propósito, se formularon los siguientes objetivos específicos:

* Diagnosticar la situación actual de la organización con respecto a la gestión docente.

* Determinar los requerimientos del sistema a partir del diagnóstico previo.

* Elaborar los esquemas lógico y físico de la base de datos a partir de las necesidades y requerimientos determinados.

IPSA SCIENTIA - Vol. $5 \mathrm{~N}^{\circ} 1-2020$ 


\section{Métodos}

A fin de describir el procedimiento seguido para alcanzar los objetivos, se seleccionó el enfoque del Ciclo de Vida del Desarrollo de Sistemas (Kendall \& Kendall, 2011), considerando aquellas fases necesarias para materializar el diseño propuesto (Linares \& Geizzelez, 2007; Gómez et al., 2013; Garcés \& Egas, 2013; Velandia \& López, 2015; Gamboa, 2018). En el cuadro 1 se detalla cada una de las actividades planificadas en función de las fases de la metodología seleccionada, a fin de dar respuesta a los diferentes objetivos planteados, junto con las técnicas y las herramientas empleadas para ello.

Cuadro 1. Actividades según la metodología

\begin{tabular}{|c|c|c|c|}
\hline Objetivo Específico & Fase de la Metodología & Actividades & $\begin{array}{c}\text { Técnicas y } \\
\text { Herramientas }\end{array}$ \\
\hline $\begin{array}{l}\text { Diagnosticar la situación } \\
\text { actual de la organización } \\
\text { con respecto a la gestión } \\
\text { docente. }\end{array}$ & $\begin{array}{l}\text { Identificación de } \\
\text { problemas, oportunidades y } \\
\text { objetivos. }\end{array}$ & $\begin{array}{l}\text { Estudio del problema mediante } \\
\text { observación. }\end{array}$ & \multirow{2}{*}{$\begin{array}{l}\text { Observación Directa. } \\
\text { Lista de requerimientos. }\end{array}$} \\
\hline \multirow{2}{*}{$\begin{array}{l}\text { Determinar los } \\
\text { requerimientos y } \\
\text { necesidades del sistema a } \\
\text { partir del diagnóstico } \\
\text { previo. }\end{array}$} & $\begin{array}{l}\text { Determinación de los } \\
\text { requerimientos de } \\
\text { información }\end{array}$ & $\begin{array}{l}\text { Elaboración del listado de } \\
\text { requerimientos. }\end{array}$ & \\
\hline & $\begin{array}{l}\text { Análisis de las necesidades } \\
\text { del sistema }\end{array}$ & $\begin{array}{l}\text { Elaboración del diagrama de } \\
\text { actividades del sistema actual. }\end{array}$ & \multirow{4}{*}{$\begin{array}{l}\text { Herramienta de diseño } \\
\text { StarUML. } \\
\text { Computador. }\end{array}$} \\
\hline \multirow{3}{*}{$\begin{array}{l}\text { Elaborar los esquemas } \\
\text { lógico y físico de la base de } \\
\text { datos a partir de las } \\
\text { necesidades y } \\
\text { requerimientos } \\
\text { determinados. }\end{array}$} & \multirow{3}{*}{$\begin{array}{l}\text { Diseño del sistema } \\
\text { recomendado }\end{array}$} & $\begin{array}{l}\text { Elaboración del diagrama de casos de } \\
\text { uso del sistema propuesto. }\end{array}$ & \\
\hline & & Diseño conceptual de la base de datos. & \\
\hline & & Diseño físico de la base de datos. & \\
\hline
\end{tabular}

Fuente: Elaboración propia

\section{Resultados}

\section{Estudio del problema mediante observación}

Con la aplicación de la técnica de observación directa se pudo identificar los problemas más resaltantes del proceso de gestión docente en la Escuela de Nutrición y Dietética de L.U.Z, dada la inexistencia de información actualizada de los profesores, horario de clases, proyectos de investigación, entre otros.

Se evidenció que existe pérdida de tiempo al momento de realizar la evaluación de cursos, errores de cálculo al procesar la información relacionada con las evaluaciones y control de asistencia suministrada por los estudiantes al profesor, así como la falta de registros que permitan realizar una comparación de la gestión docente de un semestre con respecto a otro período. 
Modelado del registro automatizado de la gestión docente para una institución universitaria

En tal sentido, la implementación de un sistema de información ofrece como oportunidades automatizar el proceso de captura de datos en el control de gestión docente, debido a que actualmente los formatos (de evaluación del curso, control de gestión docente, control de actividades académicas semestrales, informes del profesor, horarios académicos y permanencia) son llenados manualmente por los docentes de los diferentes departamentos de la escuela y luego deben ser entregados al jefe del departamento respectivo quien los presenta en la asamblea departamental para ser discutidos al comienzo de cada periodo semestral, lo que genera pérdida de tiempo y un gran número de errores de cálculo.

\section{Elaboración del listado de requerimientos}

Considerando todos los detalles anteriormente descritos, se procedió con la definición de los principales requerimientos de índole funcional que deben ser satisfechos por el sistema propuesto, los cuales se resumen en el siguiente listado:

- Control de acceso que permita el manejo de privilegios de seguridad particulares para cada usuario.

- Registro de los datos del personal docente y de investigación: nombres, apellidos, cédula de identidad, dependencia, departamento, títulos obtenidos, tipo de personal, condición, dedicación, situación, categoría, fecha de ingreso y fecha del último ascenso.

- Registro de actividades académicas: descripción y tipo de actividad (docencia, investigación, extensión, perfeccionamiento docente y administrativas), facultad, dependencia, departamento donde desempeña la actividad, así como el número de semanas y la cantidad de horas que le dedica a ellas.

- Control de actividades docentes: por cada una de las cátedras que el personal administra, debe llevarse la planificación de actividades durante el período académico actual, indicando para cada una el número de la semana, lapso, día de la semana, actividad docente cumplida y observaciones.

- Control de actividades académicas: consiste en un informe de dichas actividades y que a su vez debe contener el período, departamento y dependencia donde se realizó, su descripción, las personas participantes, fechas de inicio y finalización, número de horas programadas y cumplidas, así como ciertas observaciones.

\section{Elaboración del diagrama de actividades del sistema actual}

La figura 1 muestra el diagrama de actividades del proceso actual de gestion docente, en el que se resaltan los actores Profesor, Jefe de Departamento y Secretaria Docente. Al respecto, se observa que los profesores, al inicio de cada período académico, suministran una serie de formatos en papel de actividades de gestión docente, los cuales son revisados por el Jefe del Departamento al que se encuentran adscritos, y en asamblea son discutidos.

En caso de existir algún error (número total de horas de acuerdo a la dedicación del profesor, inconsistencias en el horario, entre otros), los formatos son devueltos al profesor para su corrección y posteriormente entregados de nuevo al Jefe del Departamento.

Este último hace una relación de informes entregados, y son llevados a la Secretaria Docente, quien los remite junto con el resto de los informes de otros departamentos a la Facultad, quien a su vez los envía a Consejo Central de Pregrado donde finalmente son archivados.

IPSA SCIENTIA - Vol. 5 No $1-2020$ 
Modelado del registro automatizado de la gestión docente para una institución universitaria

Figura 1: Diagrama de actividades del sistema actual

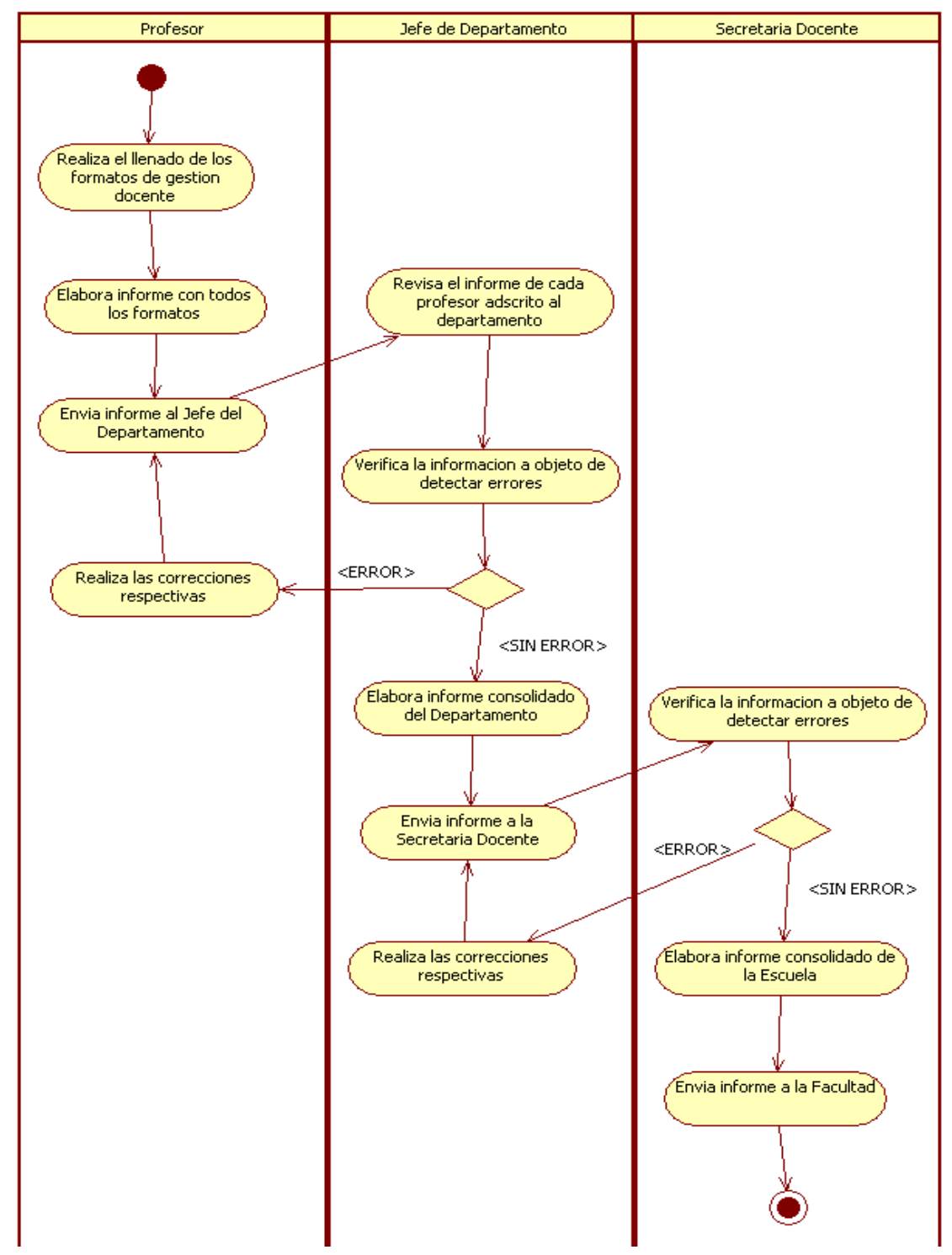

Fuente: Elaboración propia

\section{Elaboración de los diagramas de casos de uso del sistema propuesto}

En esta sección se lista uno de los casos de uso más representativos del proyecto, así como los escenarios que representan el flujo básico de eventos y otros flujos alternativos que representan alguna funcionalidad significativa dentro del sistema.

Para ello, en la figura 2 se muestra el diagrama de casos de uso del sistema propuesto, de acuerdo con los requerimientos antes identificados: 
Modelado del registro automatizado de la gestión docente para una institución universitaria

Figura 2: Diagrama de casos de uso del sistema propuesto

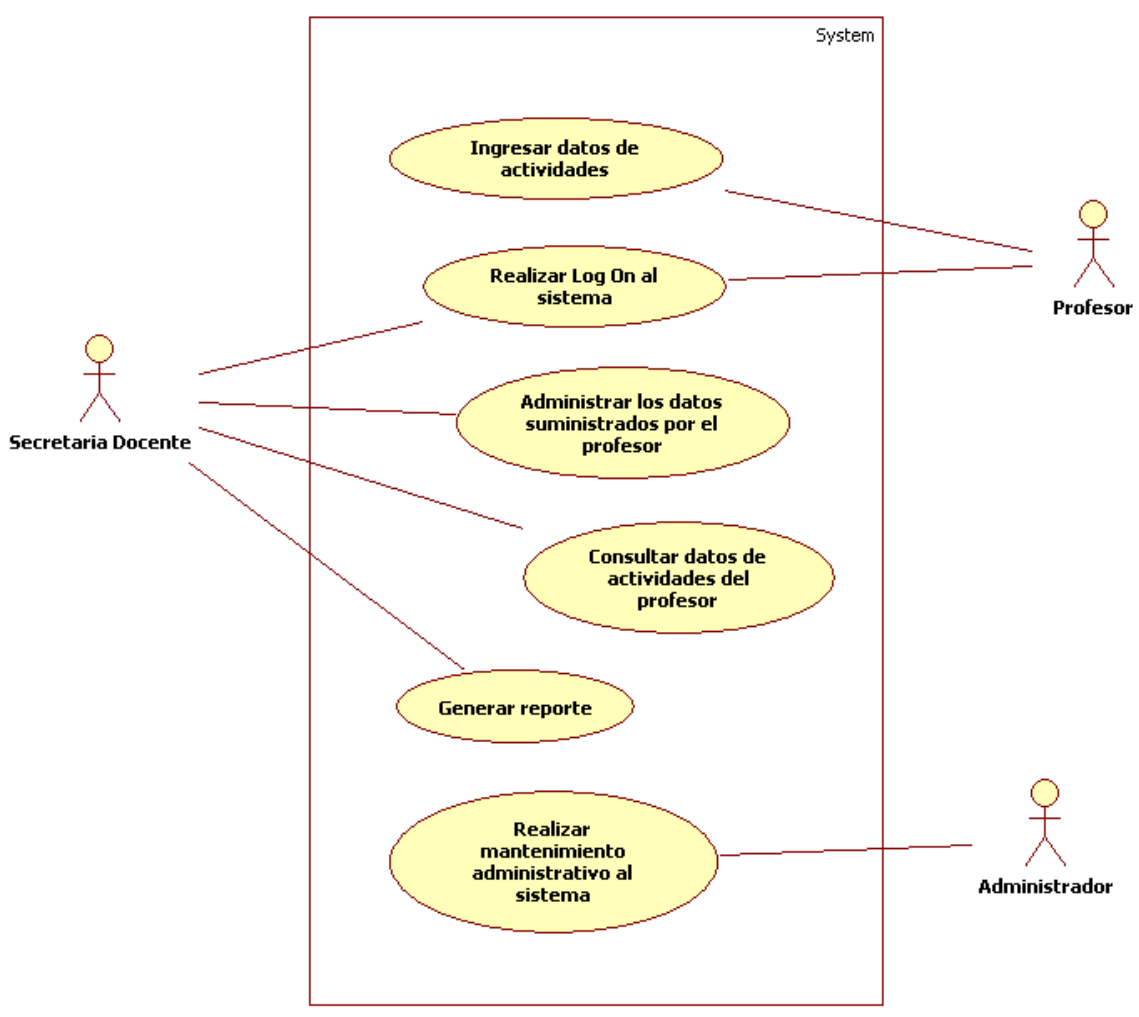

Fuente: Elaboración propia

En tal sentido, el caso de uso seleccionado corresponde a Administrar los datos suministrados por el profesor, y los escenarios abarcan el caso exitoso (Flujo Básico) y las operaciones de Insertar y Modificar (Flujos Alternativos), los cuales serán descritos más adelante.

Especificación del caso de uso seleccionado:

Nombre del Caso de Uso: Administrar los datos suministrados por el profesor.

Actores: Profesor y Secretaria Docente / Jefe del Departamento.

Descripción Breve: Este caso de uso consiste en el registro de toda la información que suministran los profesores con respecto a sus actividades académicas dentro de la Universidad.

\section{Flujo Básico de Eventos}

1. Log On.

2. Seleccionar la opción de menú: Administrar datos.

3. Ingresar un parámetro de búsqueda en el formulario: Registro de Actividades.

4. Verificar la existencia de datos cargados al evaluar el parámetro de búsqueda.

5. Mostrar resultado de la búsqueda. 
Modelado del registro automatizado de la gestión docente para una institución universitaria

Flujo Alternativo de Eventos

A1. Insertar datos nuevos.

A2. Modificar datos existentes.

A3. Eliminar datos cargados.

\section{Realización del Caso de Uso}

A continuación, se muestran los diagramas de interacción de los escenarios mencionados anteriormente. La figura 3, muestra el escenario exitoso, correspondiente al flujo básico de eventos del caso de uso Administrar los datos suministrados por el profesor:

Figura 3: Diagrama de secuencia del flujo básico

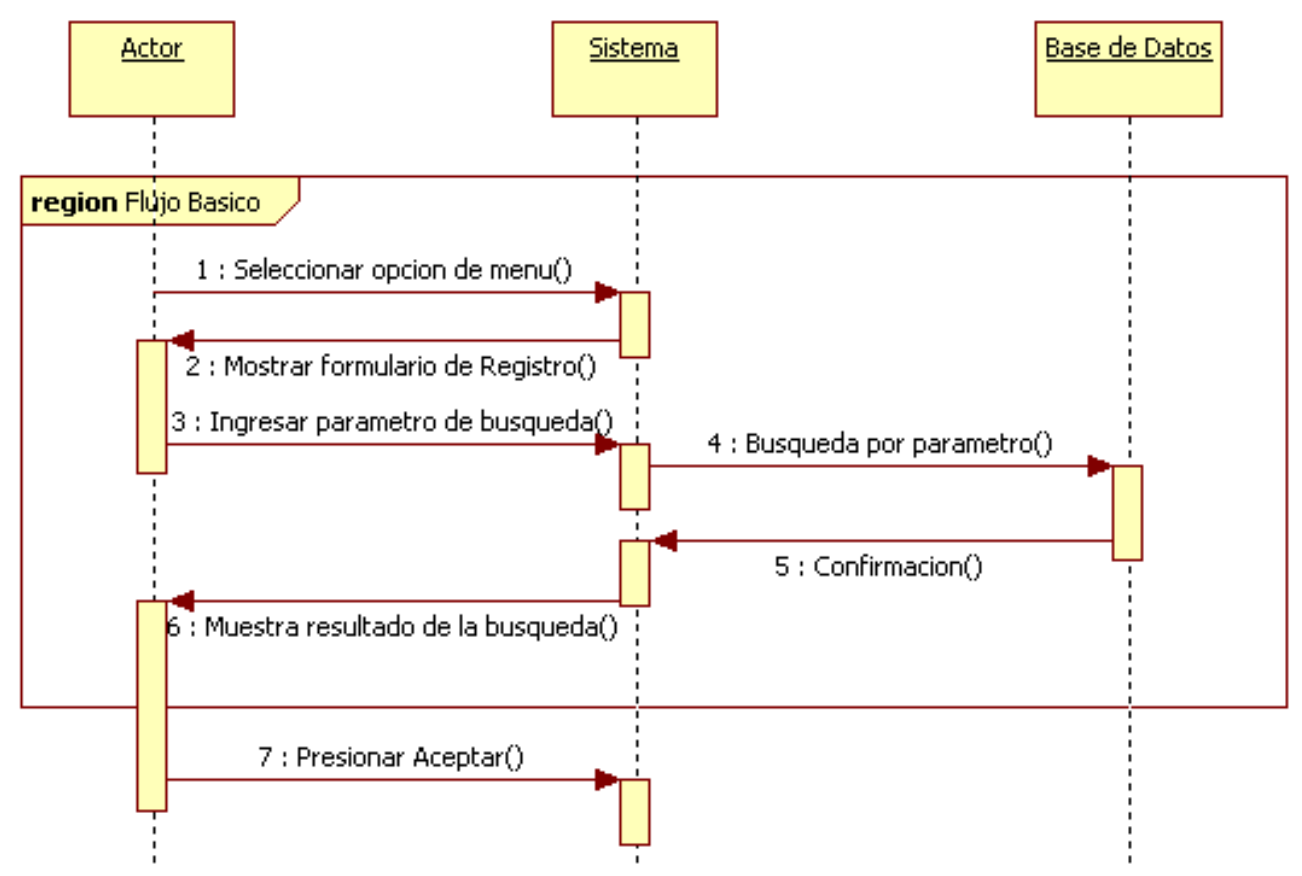

Fuente: Elaboración propia

De igual manera, el escenario alternativo de la función Insertar datos nuevos se plantea a continuación, en la figura 4:

IPSA SCIENTIA - Vol. 5 No $1-2020$ 
Modelado del registro automatizado de la gestión docente para una institución universitaria

Figura 4: Diagrama de secuencia del flujo alternativo Insertar

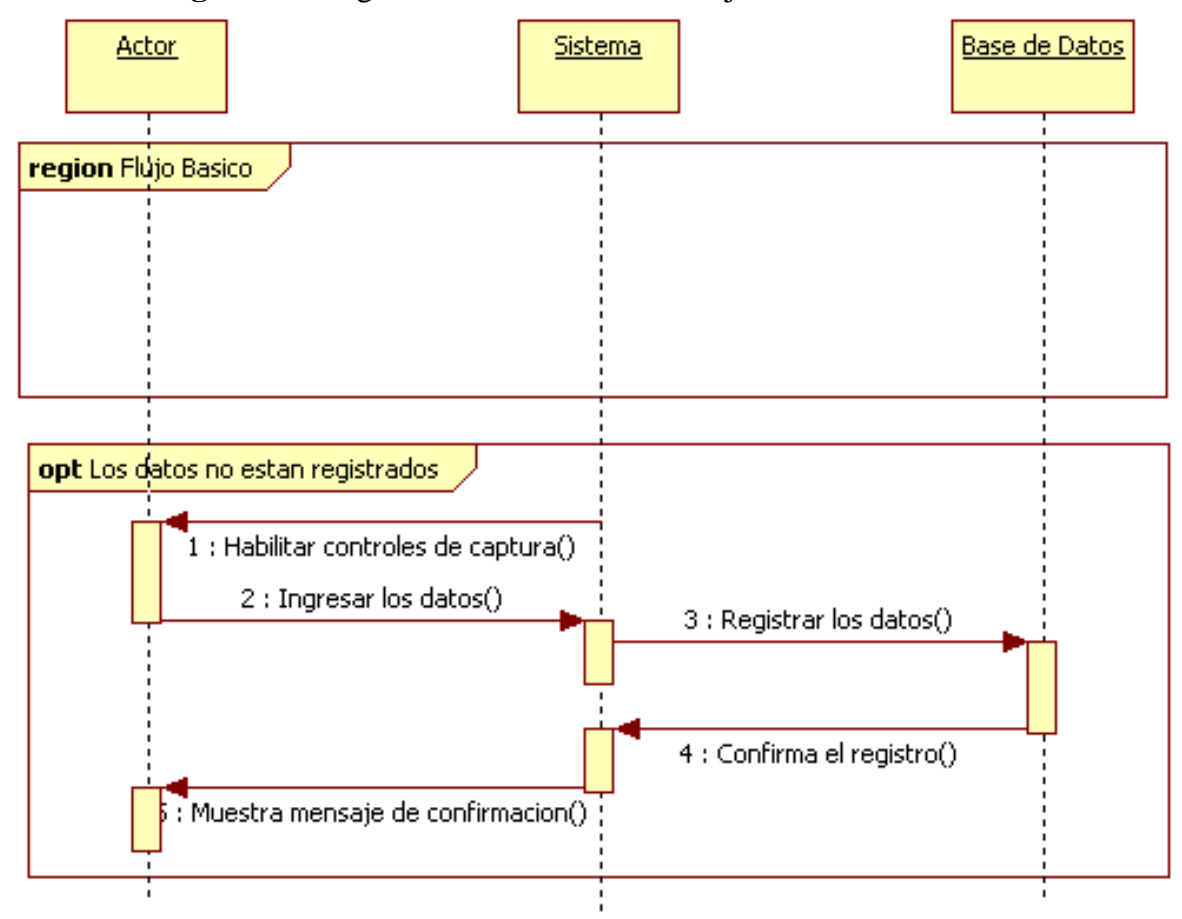

Fuente: Elaboración propia

Finalmente, el escenario alternativo de la función Modificar datos existentes se plantea en la figura 5:

Figura 5: Diagrama de secuencia del flujo alternativo Modificar

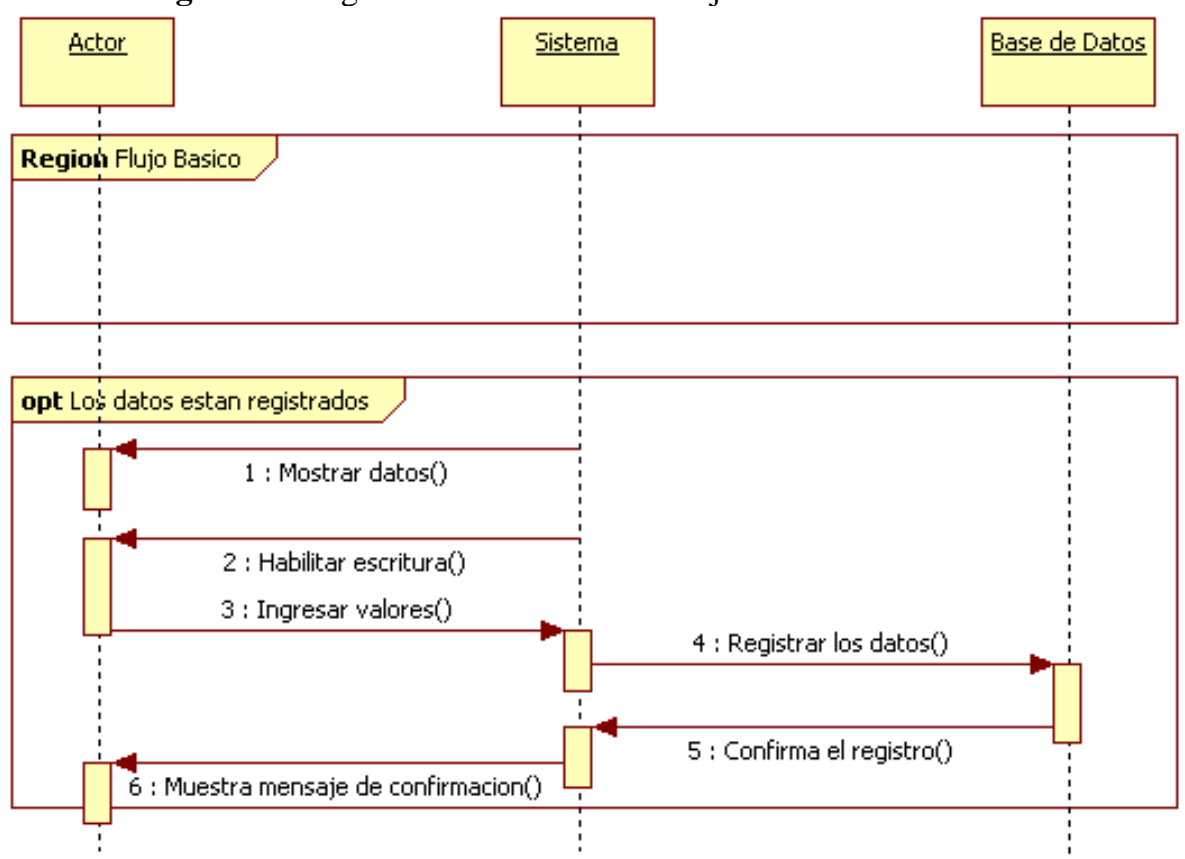

Fuente: Elaboración propia 


\section{Diseño conceptual de la base de datos}

A continuación se explican cada uno de los elementos que integran la arquitectura lógica de los datos, representado a partir de un diagrama de clases, tal como se muestra en la figura 6.

Figura 6: Diagrama de clases equivalente al modelo E-R

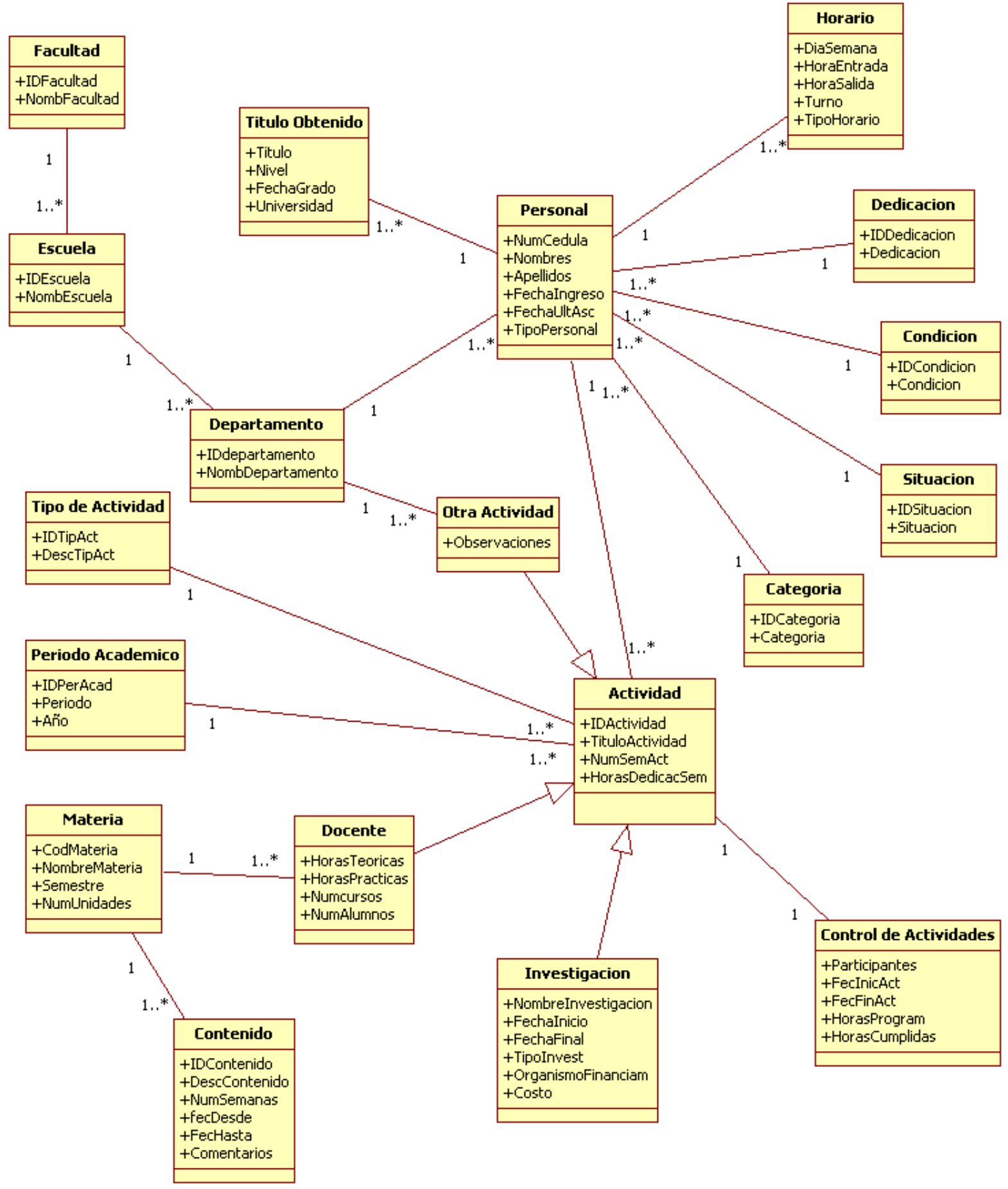

Fuente: Elaboración propia 
Modelado del registro automatizado de la gestión docente para una institución universitaria

Las clases del diagrama anterior se describen a continuación:

- Facultad: en ella se almacena el nombre de la facultad donde labora el profesor.

- Escuela: hace referencia a las diferentes escuelas que integran la facultad.

- Departamento: almacena las diferentes dependencias o departamentos que conforman la estructura organizacional de una escuela respectiva.

- Personal: en esta clase se registran los datos del personal (docente, auxiliar docente o de investigación, investigador libre u honorario) que labora dentro de la escuela.

- Título Obtenido: almacena el grado de formación académica del personal adscrito a la escuela, abarca estudios de pregrado y postgrado (especialidad, maestría o doctorado).

- Horario: se registra los diferentes bloques de hora por día de la semana, que el docente utiliza para desempeñar sus actividades académicas. Debe indicarse la hora de inicio y culminación, así como el turno correspondiente.

- Dedicación: dentro de esta clase se describe la cantidad de horas semanales de labor, a saber: dedicación exclusiva, tiempo completo, medio tiempo, tiempo convencional o especificado.

- Categoría: es aquí donde se registra la cualidad del docente y puede ser: profesor; titular, asociado, agregado, asistente, instructor o no clasificado, auxiliar docente; I, II, III, IV, V o no clasificado.

- Condición: registra la circunstancia bajo la que el docente labora, puede ser ordinario, contratado, eventual, interno, invitado, becario docente u otro.

- Situación: registra la condición actual del docente, el cual puede estar activo, becado, sabático, permiso remunerado o no, jubilado activo, jubilado, en comisión de servicio u otro.

- Actividad: esta clase hace referencia a la descripción de la actividad específica desempeñada por el personal, así como el número de semanas planificadas para cumplirla y la cantidad de horas de dedicación semanal.

- Tipo de Actividad: dentro de este objeto se almacena el tipo de actividad académica que puede realizar el docente dentro de la escuela. Existen varios tipos de actividades las cuales son: actividades docentes, de investigación, extensión, perfeccionamiento docente y actividades administrativas.

- Periodo Académico: en esta clase se registran los diferentes períodos académicos, a objeto de llevar un registro histórico por profesor de las actividades desempeñadas.

- Control de Actividades: su propósito consiste en el registro del estado de las actividades planificadas en función del tiempo, sobre la base de las fechas de inicio y finalización, el número de horas programadas y cumplidas, así como los participantes involucrados.

- Docente: representa los datos relacionados con las actividades docentes, en cuanto a número de horas teóricas y prácticas, número de cursos y alumnos inscritos por cada materia que el profesor imparte.

- Materia: constituye la clase que almacena los datos de la materia en cuestión, el nombre de la unidad curricular, el semestre en el que se ubica dentro del plan de estudios y la cantidad de unidades programadas.

- Contenido: por cada una de las materias que el profesor imparte, se registra en esta clase el contenido correspondiente al plan de estudio respectivo, indicando el número de semanas planificadas para cubrirlo y las fechas de inicio y fin del mismo.

- Investigación: en esta clase se registra las características de las investigaciones que el profesor está ejecutando durante un período académico específico, detallando el tipo de investigación, la fuente de financiamiento y el costo asociado al proyecto.

- Otra Actividad: se utiliza para representar otras actividades adicionales que puede desempeñar el profesor (perfeccionamiento docente, extensión, entre otras).

IPSA SCIENTIA - Vol. 5 No $1-2020$ 


\section{Diseño físico de la base de datos}

Finalmente, se muestran las estructuras de almacenamiento persistente de datos que constituyen el modelo de implementación de la arquitectura de datos del sistema, el cual está representado por una Base de Datos Relacional, cuyas tablas cumplen con los criterios de normalización correspondientes a la Tercera Forma Normal.

En total, son diecinueve (19) los archivos o tablas que integran la base de datos propuesta; en la mayoría de ellas se define un campo clave principal o primaria que permite la identificación de cualquier registro contenido en la tabla. De igual manera, las relaciones entre las diferentes estructuras de almacenamiento se implementan mediante la definición de campos clave foráneas a objeto de lograr vínculos de uno a varios. El detalle de cada una de las tablas se muestra en las figuras siguientes, donde se puede apreciar el nombre del campo respectivo, el tipo de dato vinculado al mismo y un breve comentario para denotar la condición de clave primaria o foránea.

Cuadro 2: Archivo Facultad

\begin{tabular}{|l|c|c|}
\hline \multicolumn{2}{|c|}{ TABLA: Facultad } \\
\hline \multicolumn{1}{|c|}{ Campo } & Tipo de Dato & Comentario \\
\hline IDFacultad & Numeric(2) & Clave Principal de la tabla. \\
\hline NombFacultad & VarChar(25) & \\
\hline
\end{tabular}

Fuente: Elaboración propia

El cuadro 2 representa la tabla Facultad, los campos corresponden al número de identificación de la facultad y su nombre. De igual manera, el cuadro 3 corresponde a la tabla Escuela, la estructura es similar a la tabla Facultad, especificando además la relación entre ellas a través del campo IDFacultad; mientras que el cuadro 4 muestra la tabla Departamento, en ella se guardan las diferentes dependencias que integran una escuela específica, por lo que se incluye el campo IDEscuela.

Cuadro 3: Archivo Escuela

\begin{tabular}{|l|c|l|}
\hline \multicolumn{2}{|c|}{ TABLA: Escuela } \\
\hline Campo & Tipo de Dato & Comentario \\
\hline IDEscuela & Numeric(2) & Clave Principal de la tabla. \\
\hline NombEscuela & VarChar(30) & \\
\hline IDFacultad & Numeric(2) & Clave Foránea (hace referencia a la tabla Facultad). \\
\hline
\end{tabular}

Fuente: Elaboración propia

Cuadro 4: Archivo Departamento

\begin{tabular}{|l|c|l|}
\hline \multicolumn{1}{|c|}{ Campo } & Tipo de Dato & \multicolumn{1}{c|}{ TABLA: Departamento } \\
\hline IDDepartamento & Numeric(2) & Clave Principal de la tabla. \\
\hline NombDepartamento & VarChar(30) & \\
\hline IDEscuela & Numeric(2) & Clave Foránea (hace referencia a la tabla Escuela). \\
\hline
\end{tabular}

Fuente: Elaboración propia

IPSA SCIENTIA - Vol. 5 No $1-2020$ 
Modelado del registro automatizado de la gestión docente para una institución universitaria

Cuadro 5: Archivo Personal

\begin{tabular}{|l|c|l|}
\hline \multicolumn{1}{|c|}{ Campo } & Tipo de Dato & \multicolumn{1}{c|}{ Comentario } \\
\hline NumCedula & VarChar(10) & Clave Principal de la tabla. \\
\hline Nombres & VarChar(30) & \\
\hline Apellidos & VarChar(30) & \\
\hline FechaIngreso & Date & \\
\hline FechaUltAsc & Date & \\
\hline TipoPersonal & VarChar(16) & \\
\hline IDDepartamento & Numeric(2) & Clave Foránea (hace referencia a la tabla Departamento). \\
\hline IDDedicacion & Numeric(2) & Clave Foránea (hace referencia a la tabla Dedicación). \\
\hline IDCondicion & Numeric(2) & Clave Foránea (hace referencia a la tabla Condición). \\
\hline IDSituacion & Numeric(2) & Clave Foránea (hace referencia a la tabla Situación). \\
\hline IDCategoria & Numeric(2) & Clave Foránea (hace referencia a la tabla Categoría). \\
\hline
\end{tabular}

Fuente: Elaboración propia

El cuadro 5 representa la tabla Personal, los campos corresponden al número de cédula, nombre completo, fechas de ingreso y último ascenso, así como la definición del tipo de personal. La información adicional se vincula con otras tablas de la base de datos, por lo que se hace referencia a distintas claves foráneas definidas más adelante. Asimismo, el cuadro 6 corresponde a la tabla Título Obtenido, la cual se vincula al archivo de Personal mediante el número de cédula y permite registrar los datos relacionados con el nivel profesional alcanzado por el docente, la universidad donde se formó y la fecha de graduación.

Cuadro 6: Archivo Título Obtenido

\begin{tabular}{|l|c|l|}
\hline \multicolumn{3}{|c|}{ TABLA: Titulo Obtenido } \\
\hline \multicolumn{1}{|c|}{ Campo } & Tipo de Dato & Comentario \\
\hline NumCedula & VarChar(10) & Clave Foránea (hace referencia a la tabla Personal). \\
\hline Titulo & VarChar(20) & \\
\hline Nivel & VarChar(15) & \\
\hline FechaGrado & Date & \\
\hline Universidad & VarChar(25) & \\
\hline
\end{tabular}

Fuente: Elaboración propia

Cuadro 7: Archivo Horario

\begin{tabular}{|l|c|c|}
\hline \multicolumn{2}{|c|}{ TABLA: Horario } \\
\hline Campo & Tipo de Dato & Comentario \\
\hline NumCedula & VarChar(10) & Clave Foránea (hace referencia a la tabla Personal). \\
\hline DiaSemana & VarChar(10) & \\
\hline HoraEntrada & Time & \\
\hline HoraSalida & Time & \\
\hline Turno & VarChar(10) & \\
\hline TipoHorario & VarChar(12) & \\
\hline
\end{tabular}

Fuente: Elaboración propia

IPSA SCIENTIA - Vol. 5 No $1-2020$ 
Modelado del registro automatizado de la gestión docente para una institución universitaria

En cuanto al cuadro 7, se tienen los datos de la tabla Horario, tales como el día de la semana donde el docente desempeña actividades académicas, la hora de entrada y salida, el turno al cual corresponde el horario, y si el mismo corresponde a su jornada académica o su permanencia. Se vincula con la tabla Personal mediante el número de cédula.

Con respecto al cuadro 8, se tiene la tabla Dedicación, los campos corresponden al número de identificación de la dedicación y su descripción. La misma estructura se tiene para las tablas Condición, Situación y Categoría, las cuales se muestran en los cuadros 9, 10 y 11 respectivamente.

Cuadro 8: Archivo Dedicación

\begin{tabular}{|l|c|l|}
\hline \multicolumn{2}{|c|}{ TABLA: Dedicación } \\
\hline \multicolumn{1}{|c|}{ Campo } & Tipo de Dato & \multicolumn{1}{c|}{ Comentario } \\
\hline IDDedicacion & Numeric(2) & Clave Principal de la tabla. \\
\hline Dedicación & VarChar(10) & \\
\hline
\end{tabular}

Fuente: Elaboración propia

Cuadro 9: Archivo Condición

\begin{tabular}{|l|c|l|}
\hline \multicolumn{2}{|c|}{ TABLA: Condición } \\
\hline \multicolumn{1}{|c|}{ Campo } & Tipo de Dato & \multicolumn{1}{c|}{ Comentario } \\
\hline IDCondicion & Numeric(2) & Clave Principal de la tabla. \\
\hline Condición & VarChar(15) & \\
\hline
\end{tabular}

Fuente: Elaboración propia

Cuadro 10: Archivo Situación

\begin{tabular}{|l|c|l|}
\hline \multicolumn{2}{|c|}{ TABLA: Situación } \\
\hline \multicolumn{1}{|c|}{ Campo } & Tipo de Dato & Comentario \\
\hline IDSituacion & Numeric(2) & Clave Principal de la tabla. \\
\hline Situación & VarChar(12) & \\
\hline
\end{tabular}

Fuente: Elaboración propia

Cuadro 11: Archivo Categoría

\begin{tabular}{|l|c|l|}
\hline \multicolumn{2}{|c|}{ TABLA: Categoría } \\
\hline \multicolumn{1}{|c|}{ Campo } & Tipo de Dato & Comentario \\
\hline IDCategoria & Numeric(2) & Clave Principal de la tabla. \\
\hline Categoría & VarChar(15) & \\
\hline
\end{tabular}

Fuente: Elaboración propia

El cuadro 12 corresponde a la tabla Período Académico, en donde se especifican los lapsos correspondientes a cada año lectivo. Por su parte, el cuadro 13 muestra los campos de la tabla Actividad. En tal sentido, se registra el nombre de la actividad, la cantidad de semanas y las horas de dedicación estipuladas. Se vincula con las tablas Personal, Actividad y Período Académico. 
Modelado del registro automatizado de la gestión docente para una institución universitaria

Cuadro 12: Archivo Período Académico

\begin{tabular}{|l|c|l|}
\hline \multicolumn{3}{|c|}{ TABLA: Período Académico } \\
\hline \multicolumn{1}{|c|}{ Campo } & Tipo de Dato & \multicolumn{1}{c|}{ Comentario } \\
\hline IDPerAcad & Numeric(2) & Clave Principal de la tabla. \\
\hline Periodo & Integer & \\
\hline Año & Integer & \\
\hline
\end{tabular}

Cuadro 13: Archivo Actividad

\begin{tabular}{|l|c|l|}
\hline \multicolumn{1}{|c|}{ Campo } & Tipo de Dato & \multicolumn{1}{c|}{ TABLA: Actividad } \\
\hline IDActividad & Numeric(2) & Clave Principal de la tabla. \\
\hline TituloActividad & VarChar(30) & \\
\hline NumSemAct & Integer & \\
\hline HorasDedicacSem & Integer & \\
\hline NumCedula & VarChar(10) & Clave Foránea (hace referencia a la tabla Personal). \\
\hline IDTipAct & Numeric(2) & Clave Foránea (hace referencia a la tabla Tipo de Actividad). \\
\hline IDPerAcad & Numeric(2) & Clave Foránea (hace referencia a la tabla Periodo Académico) \\
\hline
\end{tabular}

Fuente: Elaboración propia

Con respecto al cuadro 14, se describen el número de identificación y descripción de cada tipo de actividad que el docente puede realizar, en la tabla con el mismo nombre.

Cuadro 14: Archivo Tipo de Actividad

\begin{tabular}{|l|c|l|}
\hline \multicolumn{3}{|c|}{ TABLA: Tipo de Actividad } \\
\hline \multicolumn{1}{|c|}{ Campo } & Tipo de Dato & Comentario \\
\hline IDTipAct & Numeric(2) & Clave Principal de la tabla. \\
\hline DescTipAct & VarChar(15) & Fuente: Elaboración propia \\
\hline
\end{tabular}

Cuadro 15: Archivo Otra Actividad

\begin{tabular}{|l|c|l|}
\hline \multicolumn{1}{|c|}{ Campo } & Tipo de Dato & \multicolumn{1}{c|}{ TABLA: Otra Actividad } \\
\hline IDDepartamento & Numeric(2) & Clave Foránea (hace referencia a la tabla Departamento). \\
\hline Observaciones & Memo & \\
\hline IDActividad & Numeric(2) & Clave Principal de la tabla (heredada de la tabla Actividad). \\
\hline
\end{tabular}

Fuente: Elaboración propia

El cuadro 15 corresponde a una derivación de la tabla Actividad, llamada Otra Actividad, en la que se almacenan las observaciones que el docente quiera indicar con respecto a las actividades de extensión y de perfeccionamiento planificadas. Al ser concebida mediante herencia o especialización, esta hereda la clave 
Modelado del registro automatizado de la gestión docente para una institución universitaria

primaria de la tabla Actividad, y a su vez, se relaciona con la tabla de Departamento a través del campo IDDepartamento.

Cuadro 16: Archivo Actividad Docente

\begin{tabular}{|l|c|l|}
\hline \multicolumn{3}{|c|}{ TABLA: Actividad Docente } \\
\hline Campo & Tipo de Dato & \multicolumn{1}{c|}{ Comentario } \\
\hline IDActividad & Numeric(2) & Clave Principal de la tabla (heredada de la tabla Actividad). \\
\hline HorasTeoricas & Integer & \\
\hline HorasPracticas & Integer & \\
\hline NumCursos & Integer & \\
\hline NumAlumnos & Integer & \\
\hline CodMateria & Numeric(6) & Clave Foránea (hace referencia a la tabla Materia). \\
\hline
\end{tabular}

Fuente: Elaboración propia

Asimismo, el cuadro 16 corresponde a la segunda derivación de la tabla Actividad, llamada Actividad Docente, la cual se vincula con la tabla Materia por medio del campo CodMateria, .y que permite almacenar la cantidad de horas teóricas y prácticas, así como el número de alumnos y cursos a ser administrados para la unidad curricular en cuestión. Ahora bien, los detalles de la tabla Materia se muestran en el cuadro 17, y el temario específico de las diferentes unidades que la componen se representa en los campos de la tabla Contenido incluidos en el cuadro 18.

Cuadro 17: Archivo Materia

\begin{tabular}{|c|c|c|}
\hline \multicolumn{3}{|r|}{ TABLA: Materia } \\
\hline Campo & Tipo de Dato & Comentario \\
\hline CodMateria & Numeric(6) & Clave Principal de la tabla. \\
\hline NombreMateria & VarChar(25) & \\
\hline Semestre & Integer & \\
\hline NumUnidades & Integer & \\
\hline
\end{tabular}

Fuente: Elaboración propia

Cuadro 18: Archivo Contenido

\begin{tabular}{|c|c|c|}
\hline \multicolumn{3}{|r|}{ TABLA: Contenido } \\
\hline Campo & Tipo de Dato & Comentario \\
\hline IDContenido & Numeric(2) & Clave Principal de la tabla. \\
\hline DescContenido & Memo & \\
\hline NumSemanas & Integer & \\
\hline FecDesde & Date & \\
\hline FecHasta & Date & \\
\hline Comentarios & Memo & \\
\hline CodMateria & Numeric(6) & Clave Foránea (hace referencia a la tabla Materia). \\
\hline
\end{tabular}

Fuente: Elaboración propia 
El cuadro 19 corresponde a la tercera y última derivación de la tabla Actividad, llamada Actividad de Investigación, en la que se representan los datos del proyecto desarrollado por el profesor, su título, las fechas de inicio y culminación, el organismo que ofrece el financiamiento y el monto del mismo.

Cuadro 19: Archivo Actividad de Investigación

\begin{tabular}{|l|c|l|}
\hline \multicolumn{1}{|c|}{ Campo } & Tipo de Dato & \multicolumn{1}{c|}{ Comentario } \\
\hline IDActividad & Numeric(2) & Clave Principal de la tabla (heredada de la tabla Actividad). \\
\hline NombreInvestigacion & Memo & \\
\hline FechaInicio & Date & \\
\hline FechaFinal & Date & \\
\hline TipoInvest & VarChar(10) & \\
\hline OrganismoFinanciam & VarChar(15) & \\
\hline Costo & Money & \\
\hline
\end{tabular}

Fuente: Elaboración propia

Finalmente, el cuadro 20 muestra los datos de la tabla Control de Actividades, que incluye el número de identificación de la actividad a controlar, así como los participantes involucrados en ella, las fechas de arranque y fin pronosticadas, y la relación horas programadas y cumplidas.

Cuadro 20: Archivo Control de Actividades

\begin{tabular}{|l|c|l|}
\hline \multicolumn{3}{|c|}{ TABLA: Control de Actividades } \\
\hline Campo & Tipo de Dato & Comentario \\
\hline IDActividad & Numeric(2) & Clave Principal de la tabla. \\
\hline Participantes & Memo & \\
\hline FechaInicAct & Date & \\
\hline FechaFinAct & Date & \\
\hline HorasProgram & Integer & \\
\hline HorasCumplidas & Integer & \\
\hline
\end{tabular}

Fuente: Elaboración propia

\section{Conclusiones}

Tal como se planteó al principio del artículo, el objetivo general del presente trabajo fue el diseño de una base de datos para el registro automatizado de la información generada en el proceso de gestión docente en la Escuela de Nutrición y Dietética de LUZ.

En primera instancia, se analizó la situación actual relacionada con cada una de las actividades del proceso de control docente y se determinó la necesidad de automatizarlo a través de un sistema de información que le permita a los profesores suministrar los datos generados de las actividades y a su vez disponer de la información necesaria para realizar sus labores dentro de la institución solo con la finalidad de disminuir la cantidad de tiempo y errores en la ejecución de sus tareas. De esta necesidad surgió la propuesta de una base de datos para el registro automatizado de la gestión docente. 


\section{Modelado del registro automatizado de la gestión docente para una institución universitaria}

Con base en el análisis previo, se determinaron los requerimientos de diseño orientados esencialmente a dos grandes funcionalidades: registro y control. Al respecto, la base de datos propuesta además de cumplir con su objetivo principal que consiste en garantizar el almacenamiento confiable de datos íntegros y precisos, se encuentra estructurada y organizada de tal manera que apoya el proceso de control establecido por los actores del sistema.

Finalmente, el diseño de los esquemas lógico y físico de la base de datos ofrece respuestas satisfactorias a cada uno de los requerimientos funcionales determinados, apoyado en los diagramas de casos de uso y secuencia de eventos que explican el comportamiento de la propuesta, y el manejo de las diferentes tablas que representan el modelo de implementación de la arquitectura de datos del sistema. Las clases diseñadas permiten modelar cabalmente la realidad del dominio del problema objeto de estudio.

\section{Referencias}

Acosta, L. A., Becerra, F. A., \& Jaramillo, D. (2017). Sistema de Información Estratégica para la Gestión Universitaria en la Universidad de Otavalo (Ecuador). Formación universitaria, 10(2), 103-112.

Barrera Velasco, D. P., García Cué, J. L., Medina Ramírez, R. C., Santacruz de León, E. E., \& Escobar Aguayo, J. J. (2017). Propuesta de un sistema informático para la gestión de calidad educativa agrícola (SIGCEA). Revista mexicana de ciencias agrícolas, 8(2), 449-456.

Castillo, V. D. M., Barrios, H. G., Hernández, O. R., Paz, C. R. L., \& Rivera, I. W. (2015). SIGENU-DSS-LITE: Nuevas capacidades de integración de información docente en Instituciones de Educación Superior en Cuba. Ciencias de la Información, 46(2), 3-8.

Fong Silva, W., Taron Dunoyer, A. \& Zabaleta Mesino, R. (2019). Nuevo liderazgo organizacional para fortalecer instituciones universitarias débilmente acopladas según Weick. IPSA SCIENTIA: Revista Científica Multidisciplinaria, 4(1), 60-70. Recuperado a partir de https://latinjournal.org/index.php/ipsa/article/view/938

Gamboa, J. Z. (2018). Evolución de las Metodologías y Modelos utilizados en el Desarrollo de Software. INNOVA Research Journal, 3(10), 20-33.

Garcés, L., \& Egas, L. M. (2013). Evolución de las Metodologías de desarrollo de la Ingeniería de software en el proceso la Ingeniería de Sistemas Software. Revista Científica y Tecnológica UPSE, 1(3).

Gómez, J. M. M., Marín, M. E. H., \& Díaz, E. A. (2013). Enfoque metodológico para el diseño de interfaces durante el ciclo de vida de desarrollo de software. Revista Gti, 12(34), 59-73.

Jiménez Puñales, S., Socarras Fernández, N., Gómez González, Y., Cabrera Blanco, R., Iglesias Yera, A. E., \& Vega Betancourt, N. (2016). Sistema automatizado para mejorar la calidad docente-asistencial en la residencia de Ginecología y Obstetricia. Edumecentro, 8(1), 28-42.

Kendall, K. y Kendall, J. (2011) Análisis y Diseño de Sistemas. 8va. Edición. México; Pearson Educación.

Linares, J. \& Geizzelez, M. (2007). Administración de proyectos en ingeniería del software. TELOS, Revista de estudios interdisciplinarios en ciencias sociales, 9(1): 26-41.

Linares Morales, J., \& Geizzelez Luzardo, M. (2016). Políticas científicas y tecnológicas para la gestión del conocimiento en instituciones universitarias. IPSA SCIENTIA: Revista Científica Multidisciplinaria, 1(1), 10-17. Recuperado a partir de https://latinjournal.org/index.php/ipsa/article/view/889

IPSA SCIENTIA - Vol. $5 \mathrm{~N}^{\circ} 1-2020$ 


\section{Modelado del registro automatizado de la gestión docente para una institución universitaria}

Miranda Cairo, M., Valdés Puga, O., Pérez Mallea, I., Portelles Cobas, R., \& Sánchez Zequeira, R. (2016). Metodología para la implementación de la Gestión Automatizada de Controles de Seguridad Informática. Revista Cubana de Ciencias Informáticas, 10(2), 14-26.

Morell González, L. M., Fernández Velázquez, L., \& Cedeño Zambrano, R. M. (2017). El sistema de control interno en los departamentos docentes: una vía para propiciar la sostenibilidad en la gestión universitaria. Cofin Habana, 11(2), 295-310.

Romero, S., \& Quintero, J. (2014). Nivel de conocimiento en programación y el desarrollo de sistemas de información automatizados/ Revista Internacional de Tecnología, Ciencia y Sociedad, 3(2).

Santana Espinosa, M. C., Muñoz Morejón, M., O'Farril Fernández, M. F., Martínez Delgado, D. A., \& Martínez Noa, M. (2017). Sistema informático para la gestión de datos del docente. Educación Médica Superior, 31(1), 89-98.

Tigre, Á. E. F., Delgado, O. W. M., \& Gavilanes, J. V. S. (2016). Consideraciones generales acerca de la gestión educativa. Dominio de las Ciencias, 2(4), 179-190.

Velandia, L. N. M., \& López, W. M. L. (2015). Escoger una metodología para desarrollar software, difícil decisión. Revista Educación en Ingeniería, 10(20), 98-109. 